\title{
TECHNIQUES FOR GAMMA RAYS
}

\author{
CARL E. FICHTEL \\ NASA/Goddard Space Flight Center, Greenbelt, Md., U.S.A.
}

\begin{abstract}
The detecting systems used in high energy astrophysics are generally more similar to particle detectors than to optical devices. The basic design of the gamma ray instrument depends on whether the energy range is below about $10 \mathrm{MeV}$ and therefore in the region where the Compton effect predominates in the absorption of the gamma-rays, or above that energy where electron-positron pair production is most important. The most usual approach to the detector system in the lower of the two energy intervals is to use a scintillation counter in the center of the detector system to absorb the photons and permit a measure of their energy, and to surround it by another detector which is employed as an active anticoincidence shield to discriminate against charged particles. In the gamma-ray interval above about $10 \mathrm{MeV}$, the very low flux of gamma rays and the high particle background has directed the development of high energy gamma-ray telescopes towards complicated techniques and large detector arrays. As a result, several investigators have now turned to the spark chamber as the heart of a detector system. Generally, it is surrounded by an anticoincidence system and is triggered by a counter telescope.
\end{abstract}

As Dr Clark has indicated in his talk, physicists have recently made an increasingly great effort to detect the highest energy photons (those with energies exceeding a few tenths of a $\mathrm{MeV}$ ) arriving from space. The study of these quanta is necessarily a very new field of astrophysics because the short path length of these photons in air generally demands that the observing instrument be placed above most of the atmosphere. Accordingly, the majority of our information about gamma rays has come from a variety of detectors flown on high altitude balloons (typically at 130000 to $140000 \mathrm{ft}$ above the surface of the Earth) and from small satellite experiments. In this talk, I shall review the types of instruments that have been used in the past and then describe those which will be flown in the future or at least are being proposed. I wish first to thank all of those who have sent me prints of their experiments, and also to express regret that time limitations will not permit me to mention all of the experiments in the field.

The detecting systems used in high energy astrophysics are generally more similar to particle detectors than to optical devices. The high frequency of the radiation precludes the use of reflection or diffraction techniques; however, the high energy content of each photon does enable them to be detected with scintillators, proportional counters, and solid state detectors. The basic design of the space gamma-ray instrument depends on whether the energy range is below about $10 \mathrm{MeV}$ and therefore in the region where the Compton effect predominates in the absorption of the gamma ray, or above that energy where electron-positron pair production is most important. Figure 1 shows the cross section for gamma rays as a function of energy.

The most usual approach to the detector system in the lower of the two energy intervals is to use a scintillation counter, which absorbs the photons and permits a measure of their energy. This counter is surrounded by another detector which is employed as an active anticoincidence shield to discriminate against charged particles, by providing the information that a charged particle has just passed through the 
system and therefore the central scintillator has not recorded an X-ray pulse. The first successful detection of gamma rays in this energy range was accomplished by Arnold and coworkers (Arnold et al., 1962; Metzger et al., 1964) using a cesium iodide crystal surrounded by a plastic scintillator. In this experiment both detectors were viewed with a single photomultiplier; discrimination between pulses in the two materials was accomplished by taking advantage of the difference in scintillation

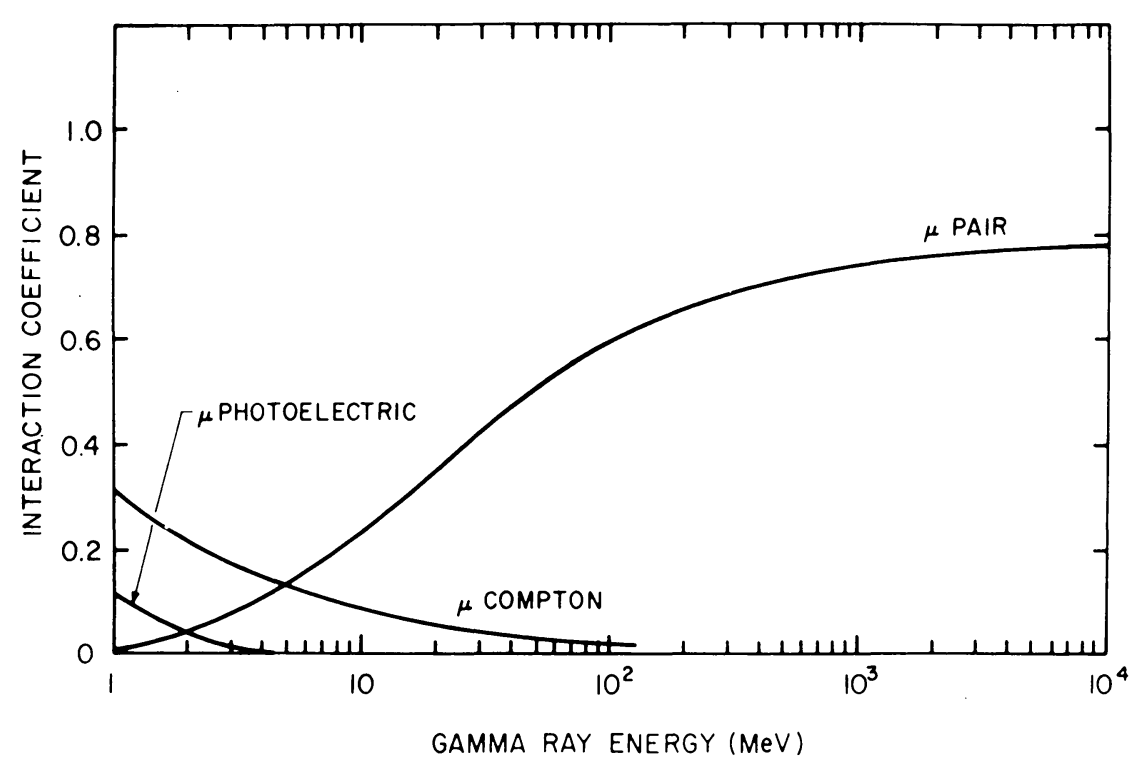

Fig. 1. Interaction coefficient for gamma rays as a function of energy for lead. The point where the Compton curve crosses the pair production curve depends somewhat on the material.

periods (about $1 \mu \mathrm{s}$ in cesium iodide and two orders of magnitude smaller in plastic scintillator). This instrument covered the range from 0.1 to $1 \mathrm{MeV}$ and was omnidirectional. Recently data has been obtained on the galactic background radiation from 0.25 to $6 \mathrm{MeV}$ using the detector shown in Figure 2 flown on ERS-18 (Peterson et al., 1968). It consists of a $7.65 \mathrm{~cm}$ long $\times 6.35 \mathrm{~cm}$ diameter $\mathrm{NaI}(\mathrm{Tl})$ scintillation counter with a $1 \mathrm{~cm}$ thick plastic anticoincidence shield. There are six channels of pulse height analysis. The anticoincidence scintillator is commutated on and off every $500 \mathrm{~s}$. The two scintillators are optically separate.

An instrument which has some directionality has been developed by both the Goddard Space Flight Center group under K. Frost and the University of California at San Diego under L. Peterson. In this detector system a thick collimating wall made of both active and passive material surrounds a central crystal. An example of this type of instrument designed by Frost and Rothe (1963) and flown on OSO is shown in Figure 3. It is sensitive in the energy region from 0.1 to $3 \mathrm{MeV}$. The large cylinder which is $7 \frac{1}{4} \mathrm{in}$. long and viewed by 4 tubes, is an anticoincidence shield for discrimination 


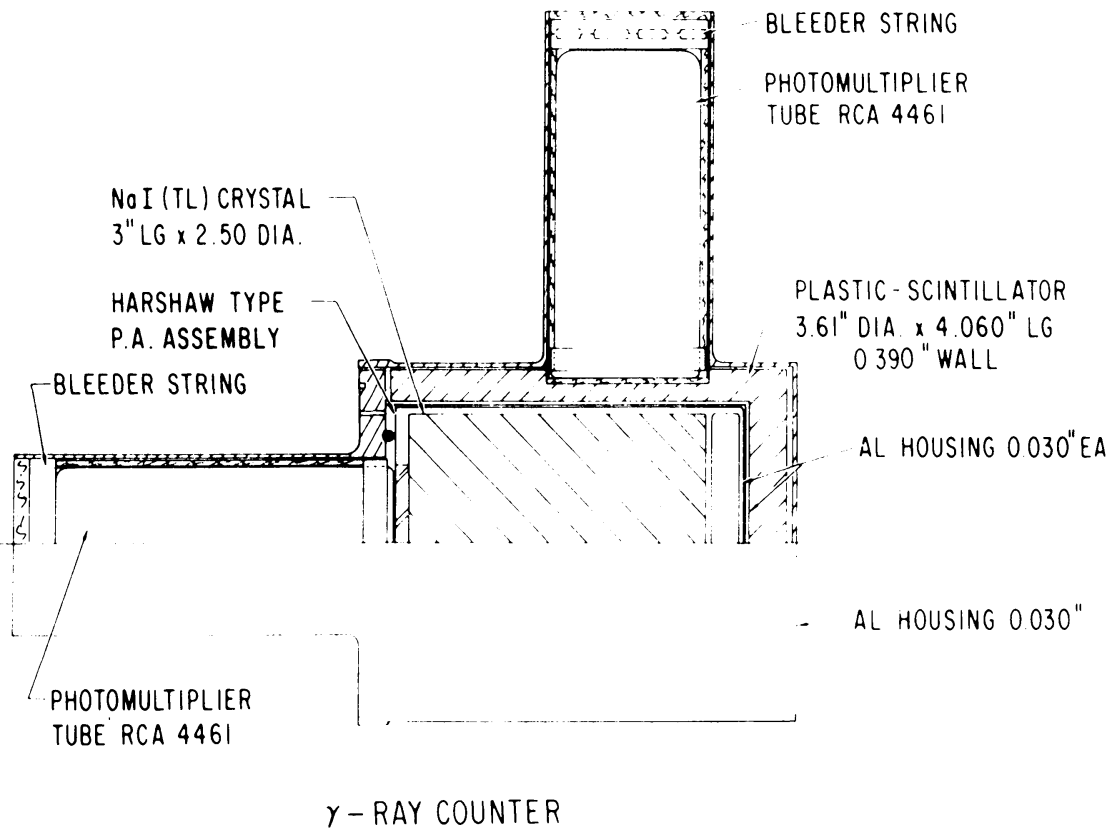

Fig. 2. Low energy gamma ray detector $(0.25$ to $6 \mathrm{MeV})$ flown by Vette, Gruber, Matteson, and Peterson on ERS-18.
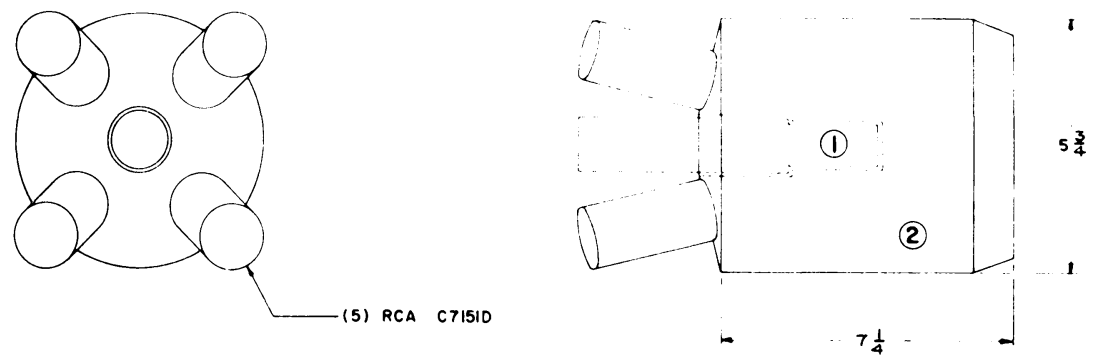

COLLIMATED GAMMA-RAY SCINTILLATION SPECTROMETER FOR ENERGY RANGE 0.1 TO $3 \mathrm{MeV}$

(1) CENTRAL SPECTROMETER CRYSTAL I" DIAMETER X 2 " LONG CS I (TI).

(2) ANTI-COINCIDENCE SHIELD FOR DISCRIMINATION AGAINST GAMMA-RAYS AND CHARGED PARTICLES AND SUPPRESSION OF COMPTON CONTINUUM.

Fig. 3. Low energy gamma ray detector designed by Frost and Rothe flown on OSO-3. 
against gamma rays and charged particles and for suppression of the Compton continuum. As its heart is a spectrometer crystal one inch in diameter and two inches long, made of thallium-doped cesium iodide. It is viewed by an RCA C7151D photomultiplier. This basic approach has also been used by Haymes and co-workers (Haymes et al., 1968a; Haymes et al. 1968b) at Rice in the study of spectra from several source regions. Figures 4 and 5 show two recent detector systems of this type and Figure 6

\section{3-10 MEV TELESCOPE}
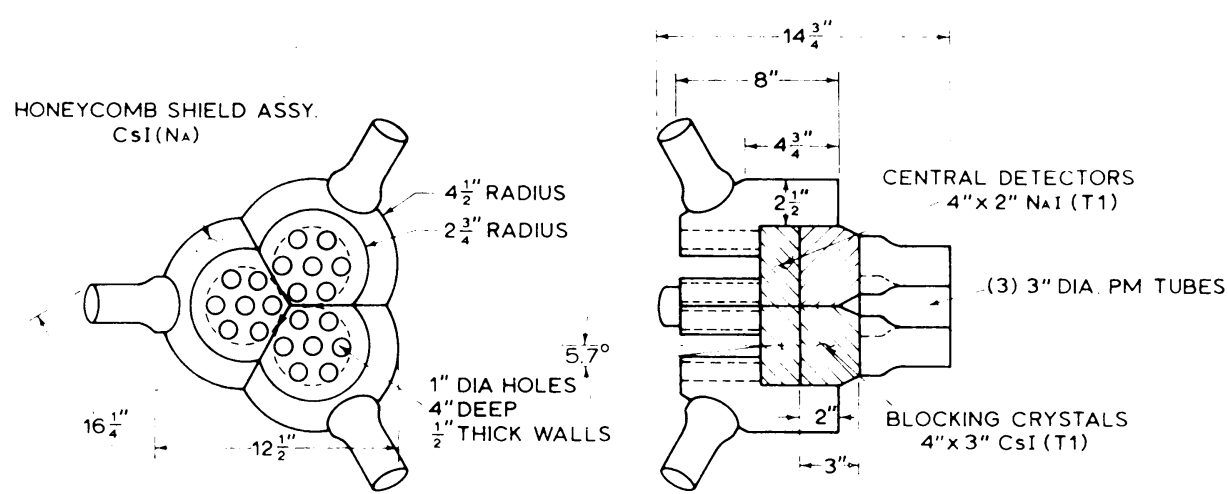

AREA $107 \mathrm{~cm}^{2}$

HALF ANGLE $5.7^{\circ}$

SOLID ANGLE $3 \times 10^{-2}$ STER

TELESCOPE FACTOR $3.21 \mathrm{~cm}^{2}-\mathrm{STER}$

CRYSTALS WEIGH 140 POUNDS

Fig. 4. A current low energy gamma ray detector being developed by Peterson and co-workers.

shows the expected sensitivity for one of them. Other detector systems to study this energy range are discussed by Bui-Van and Vedrenne (1970); Albernhe et al. (1970); and Dean et al. (1970).

The energy range from 1 to $10 \mathrm{MeV}$ remains a particularly difficult one. The combination of the low conversion cross section and the nature of the Compton reaction make directional and energy measurements extremely difficult. There appear now to be two possible directions in which experiments may go in the future. One is a small omnidirectional detector placed as far away from other matter as possible to measure the energy spectrum of the diffuse radiation as well as possible. Another is an assembly of a large number of the cylindrical collimators in an effort to increase the sensitivity and yet obtain some angular information.

Before leaving the low energy gamma region, I should perhaps mention two approaches which have not been too fruitful. In general, passive collimating devices made of lead or other material by themselves have proved to be a net disadvantage because of the creation of secondary gamma rays in the collimator. Another detector system which has not been very successful is the Compton telescope which consists of two 
separate scintillators placed in coincidence and surrounded by anti-coincidence plastic scintillators. The basic difficulties are background and low detection efficiency.

Balloon observations have generally been made either by mounting the detector system in a gondola which orients the detector as desired or by mounting the detector in a vertical position and letting the source pass overhead. Orientation information has

\section{OSO-I (EYE) 10 KEV - 1 MEV X-RAY DETECTOR}

\begin{tabular}{|lr|}
\hline \multicolumn{2}{|l|}{ PARAMETERS OF DETECTOR } \\
\hline SENSITIVE AREA & $56.9 \mathrm{~cm}^{2}$ \\
MAX. ACCEPTANCE ANGLE & $6.3^{\circ}$ \\
F W H M & $5.1^{\circ}$ \\
AREA XSOLID ANGLE FACTOR & $0.54 \mathrm{~cm}^{2} \mathrm{st}$ \\
WEIGHT OF CRYSTALS & $70 \mathrm{lbs}$ \\
\hline
\end{tabular}
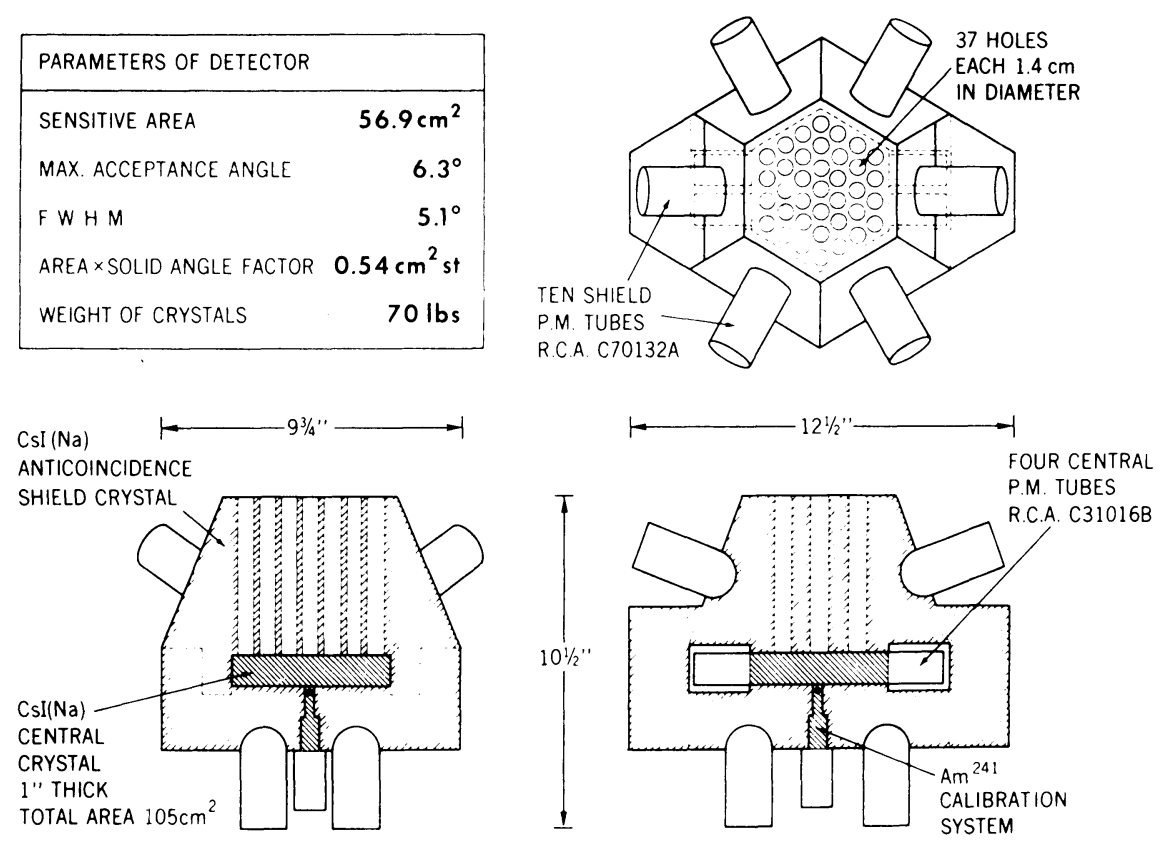

Fig. 5. A low energy gamma ray detector to be flown on OSO-1 by Frost and co-workers.

normally been obtained with the use of magnetometers and balloon position information. Figure 7 shows the balloon gondola system of Haymes at Rice.

In the gamma ray interval above about $10 \mathrm{MeV}$ the only efficient interaction process for gamma rays is the creation of a negatron-positron pair. Information on the direction and energy of the gamma ray must be derived from this pair because the penetration power of gamma rays and their low flux relative to charged particles and other radiation make shielding difficult and generally undesirable. Early experiments using counter systems and nuclear emulsions flown on balloons detected no celestial point sources of gamma rays above the background. More significant upper limits to possible fluxes from point sources, and an upper limit to the galactic flux, were obtained by Kraushaar and Clark (1962) and Kraushaar et al. (1965) using the scintillatorCerenkov type detector system shown in Figure 8 flown on Explorer 11. An improved version of the detector was flown on OSO-3 and led to the first certain measurements 
of celestial gamma rays. The detector of Clark et al. (1968) flown on OSO-3 is shown in Figure 9. The top sandwich of crystal scintillators acts as both a converter of gamma rays and as part of the telescope defining the solid angle of the incident gamma ray. The other half of the telescope is the lucite Cerenkov detector, which responds to the

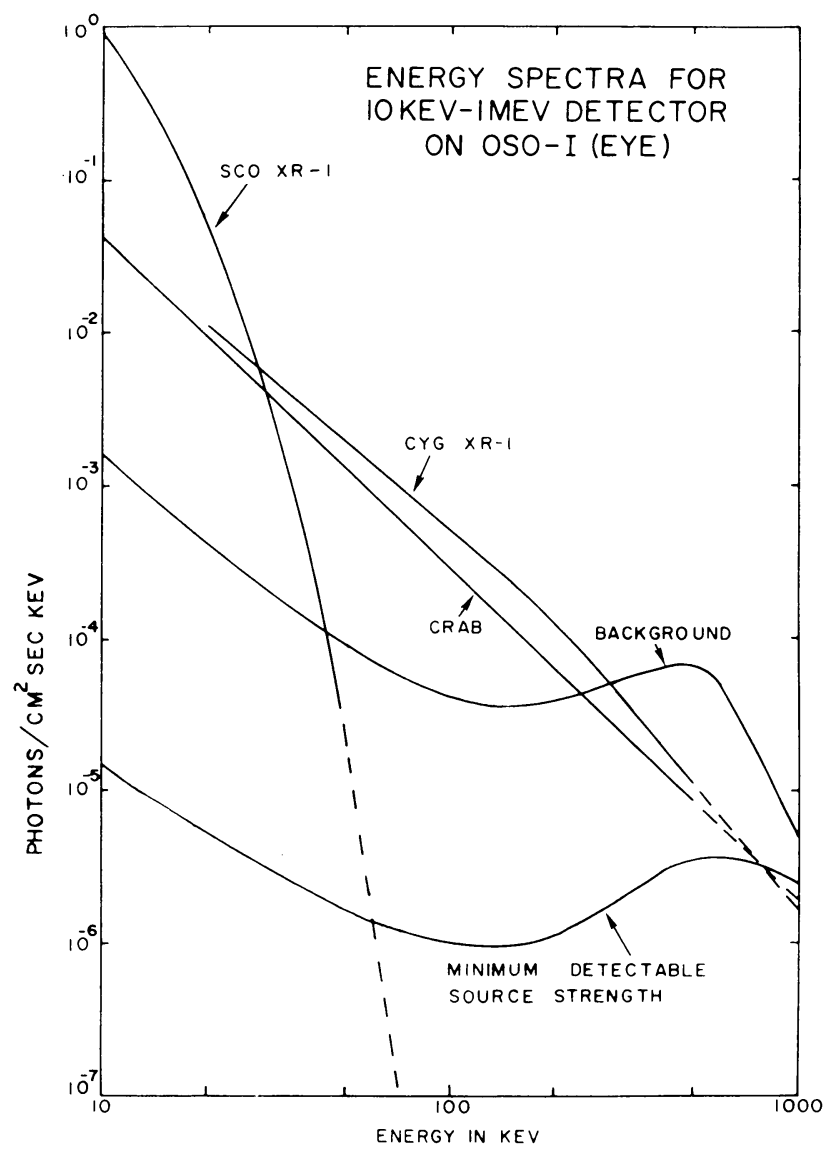

Fig. 6. Sensitivity of the OSO-1 experiment shown in the previous figure as a function of energy compared to the X-ray flux from representative sources.

Cerenkov light produced by one or both of the negatron-positron pair particles produced in the converter, and is in coincidence with the scintillator sandwich. The detector system is surrounded by a large very efficient anticoincidence dome to reject charged particles.

A scintillator-Cerenkov counter gamma ray telescope has also been flown on two satellites, Proton 2 and Cosmos 208, by Bratolyubova-Tsulukidze et al. (1969), who reported results at the Budapest conference last summer. They used a lead glass Cerenkov counter, and with the aid of pulse height analysis, divided their results into 


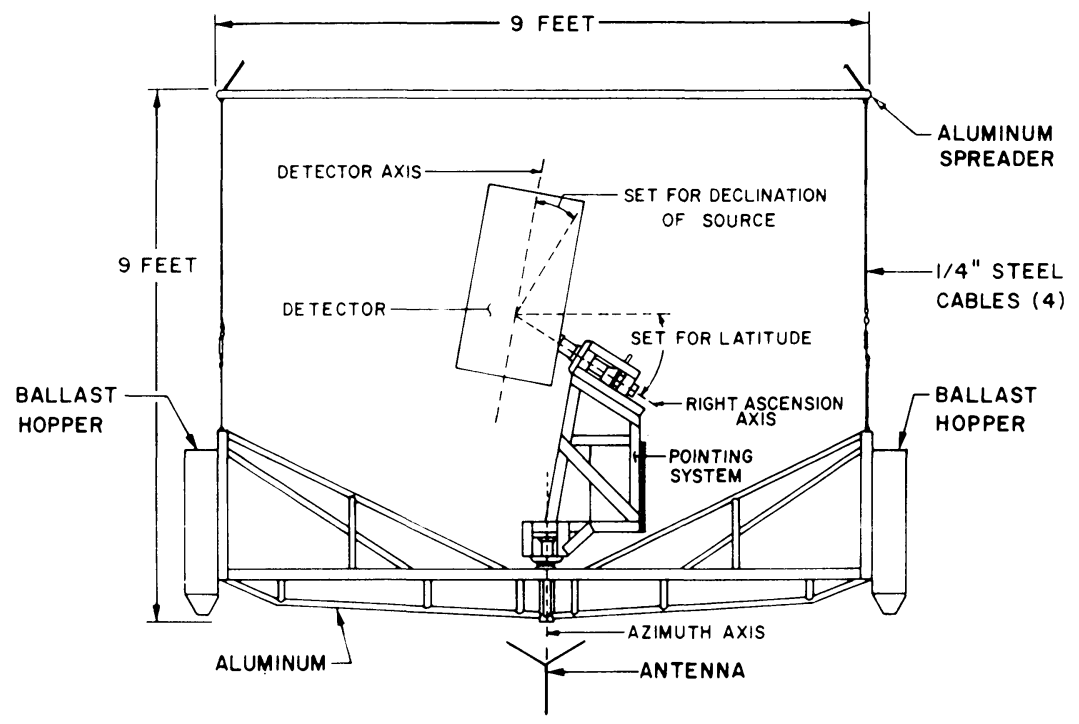

Fig. 7. Balloon orientation system used by Haymes and co-workers at Rice University.

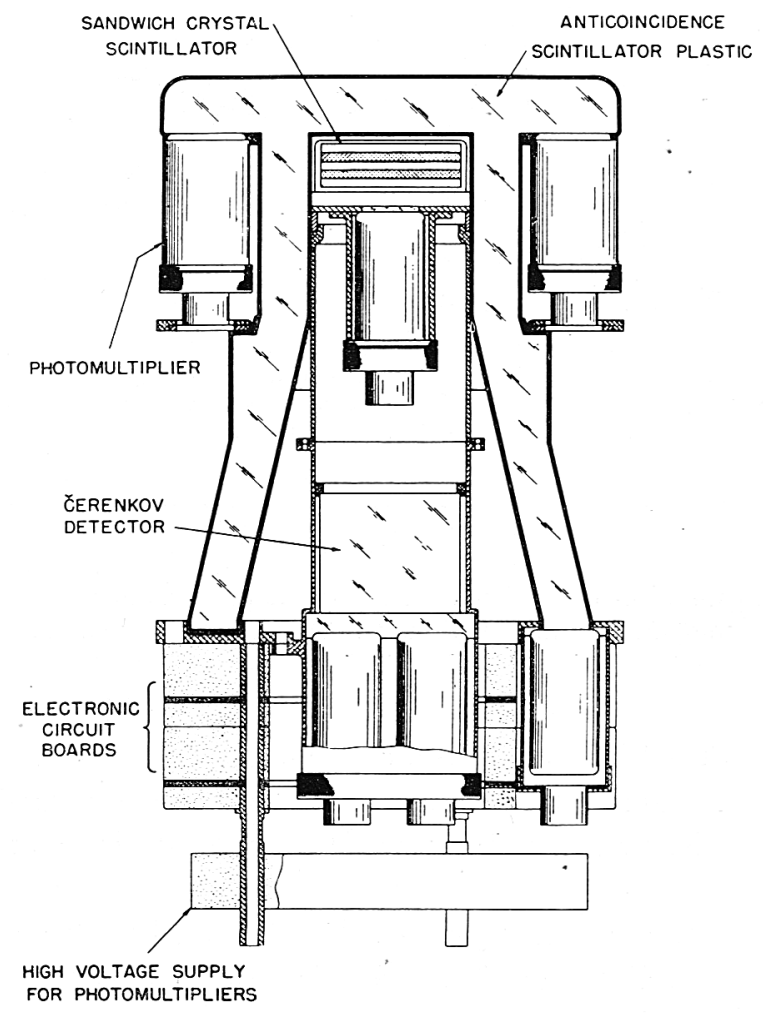

Fig. 8. High energy scintillator-Čerenkov detector flown in Explorer 11 by Kraushaar and co-workers. 
three energy bins. Other scintillator-Čerenkov gamma ray telescopes have been flown on OSO-1 by Fazio and Hafner (1967) and on OSO-3 by Kaplon and Valentine (1970) from the University of Rochester.

In view of the very low flux of gamma rays and the very high background of charged particles, it has become apparent that more complicated techniques using picture type detectors and large areas were desirable to see gamma ray point sources. Several investigators have now turned to the spark chamber as the heart of a detector system.

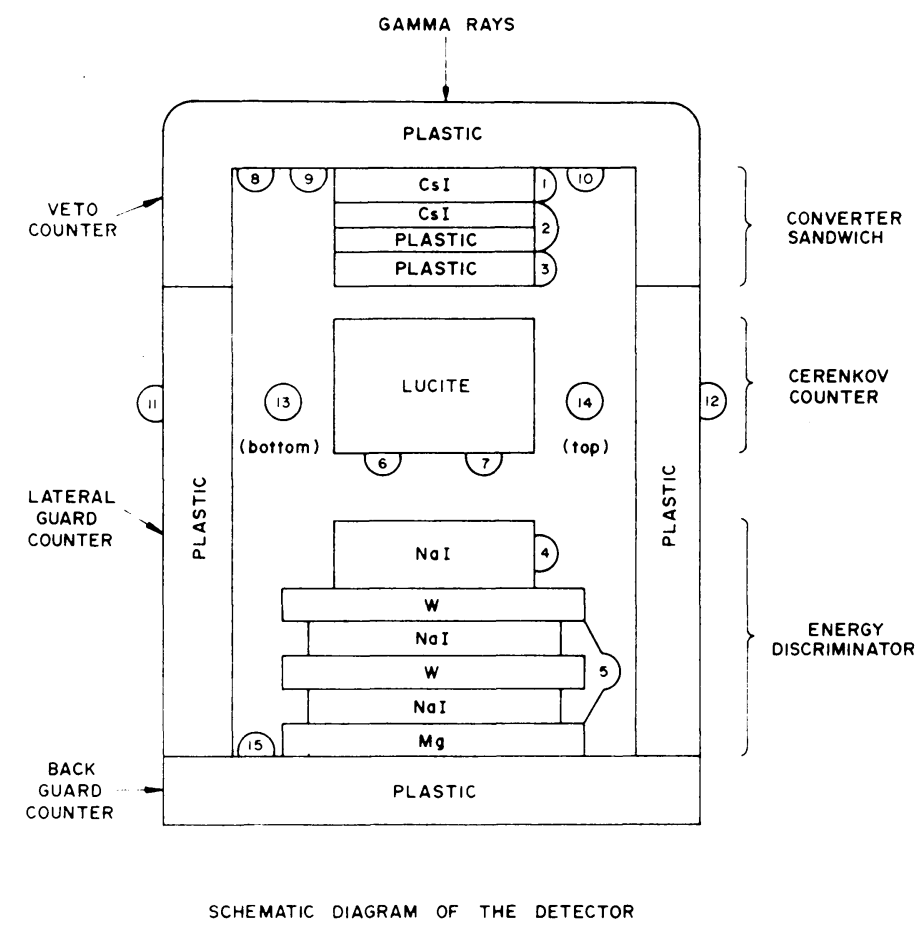

Fig. 9. High energy gamma ray detector flown by Clark, Garmire, and Kraushaar on OSO-3. This instrument led to the first certain measurement of gamma rays.

The spark chamber provides a high discrimination picture type device. An individual deck of the spark chamber detector consists of a series of parallel planes with a gas in between. A high voltage difference may be applied to pairs of these planes when a particle passes through the chamber. The voltage causes a breakdown along the ionized path left by the charged particle, and a spark forms where the particle has just passed through the chamber. The spark chamber has the advantage of being a high information content device which allows the experimenter to separate the negatronpositron pairs from the other events produced by neutral particles. Generally, the spark chamber assembly is surrounded by an anticoincidence system, and is triggered by a scintillator Cerenkov counter telescope coincidence signal and an anticoincidence signal from the surrounding anticoincidence detector. Figure 10 shows an 
example of an early version of this type of detector system showing the general configuration (Ehrmann et al., 1967).

Several different types of spark chambers have currently been developed and flown on high altitude balloons. The high altitude thin (typically less than a thousandth of an inch) polyethylene balloon of large dimensions (ten to thirty million cubic feet in

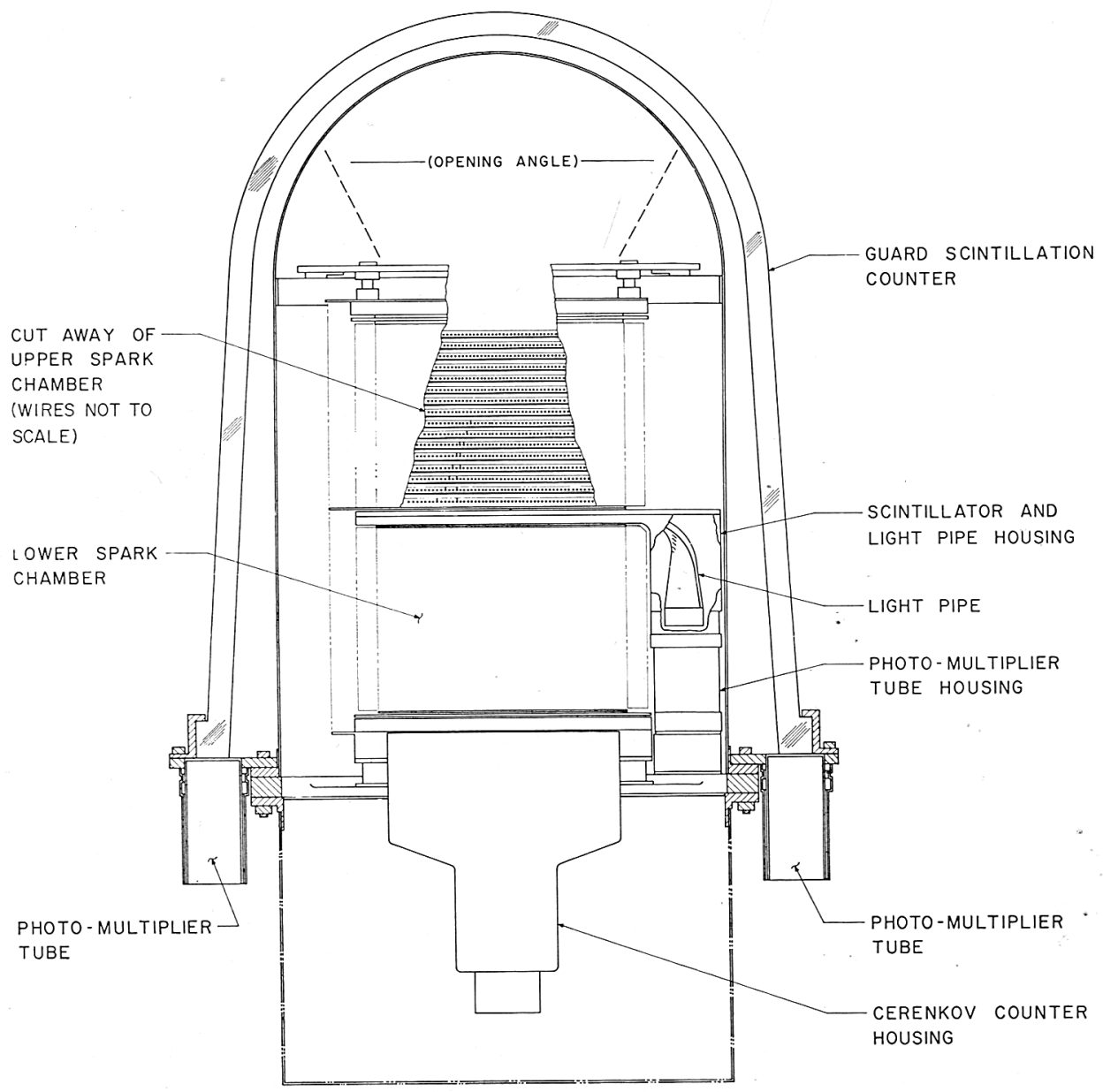

Fig. 10. Schematic diagram of the original small version of the Goddard digitized spark chamber gamma ray telescope.

volume when fully inflated at ceiling) has proved a great asset to gamma ray astronomy in providing a relatively inexpensive way to get above most of the atmosphere and to test detectors in an environment similar to space. Aspect information to $\pm \frac{1}{2}^{\circ}$ is normally available and in some cases orientation systems are used. Flight durations of from 6 to $24 \mathrm{~h}$ at ceiling are normal. Figures 11 and 12 show a balloon just before launch and during ascent. 


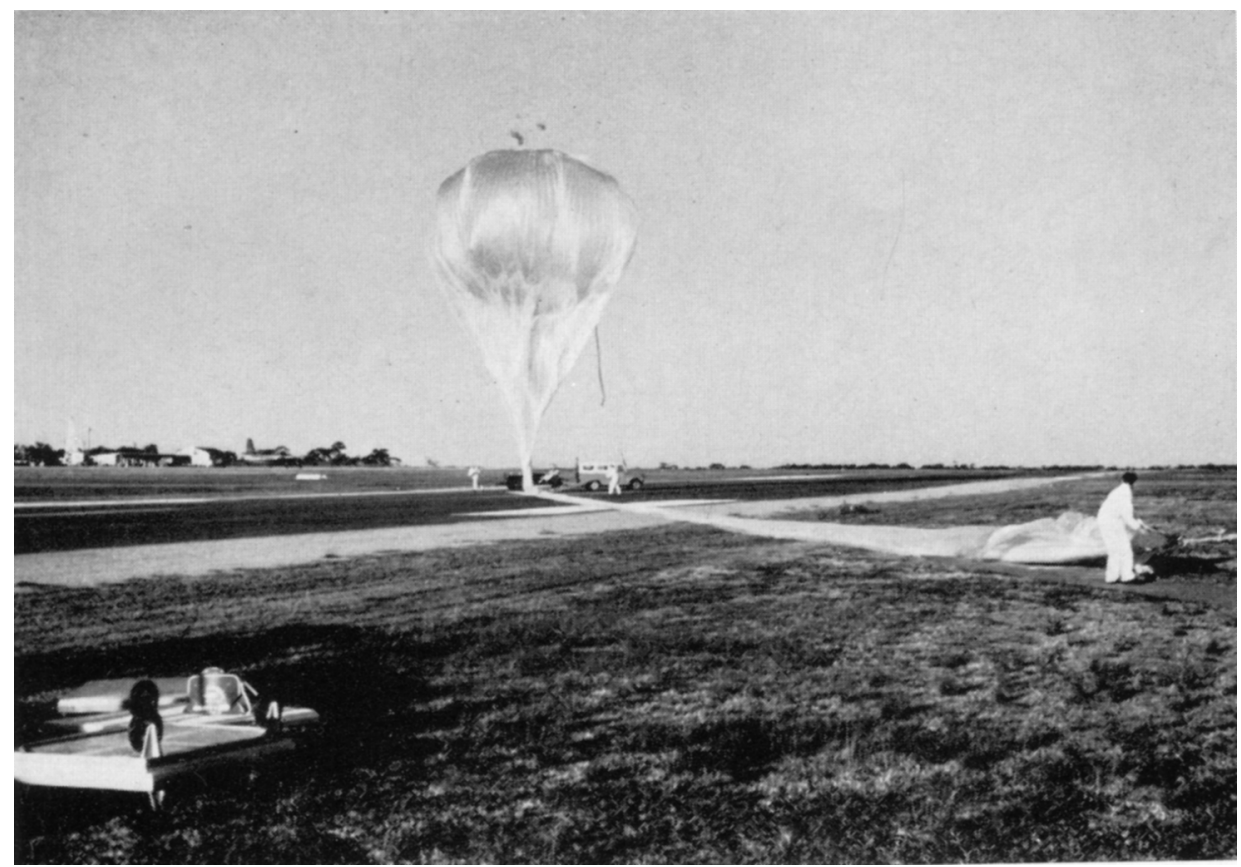

Fig. 11. A balloon just before release at Mildura, Victoria, Australia.

Four distinctly different types of spark chambers are currently being considered: the conventional optical spark chamber, the vidicon system, the sonic chamber, and the multiwire digitized spark chamber. I shall discuss all of these briefly.

The optical chamber records on film two orthogonal views of the sparks produced along the path of the negatron and positron. An example of this type of detector system developed by Frye and associates at Case Institute is shown in Figure 13 (Frye and Smith, 1966). The general approach is seen to be similar to the spark chamber telescope described earlier. Optical spark chambers have also been flown by several other groups including the one of Southampton (S. J. Board et al., 1968). The instrument of this group, shown in Figure 14, is one of the largest flown on balloons thus far having a sensitive area of about $3600 \mathrm{~cm}^{2}$. It now has 70 plates each of .01 radiation lengths. Others include those of Cobb et al. (1965) at Rochester, and Niel et al. (1969) of France.

The vidicon system developed for TD-1, and also by Fazio and coworkers for balloons (Helmken and Fazio, 1966; Fazio et al., 1968) replaces the film with a vidicon tube which then records the spark 'picture' electronically and the information may be stored on tape or telemetered. The TD-1 program is a joint effort of three institutions under the direction of Pinkau and Sommer at the Max-Planck-Institut für Extraterrestrische Physik, Koechlin at Saclay, and Boella at the University of Milan. A schematic diagram of the experiment is shown in Figure 15. The gamma ray telescope has an active area of $130 \mathrm{~cm}^{2}$ a solid angle of $0.22 \mathrm{sr}$, and an asymptotic detection 


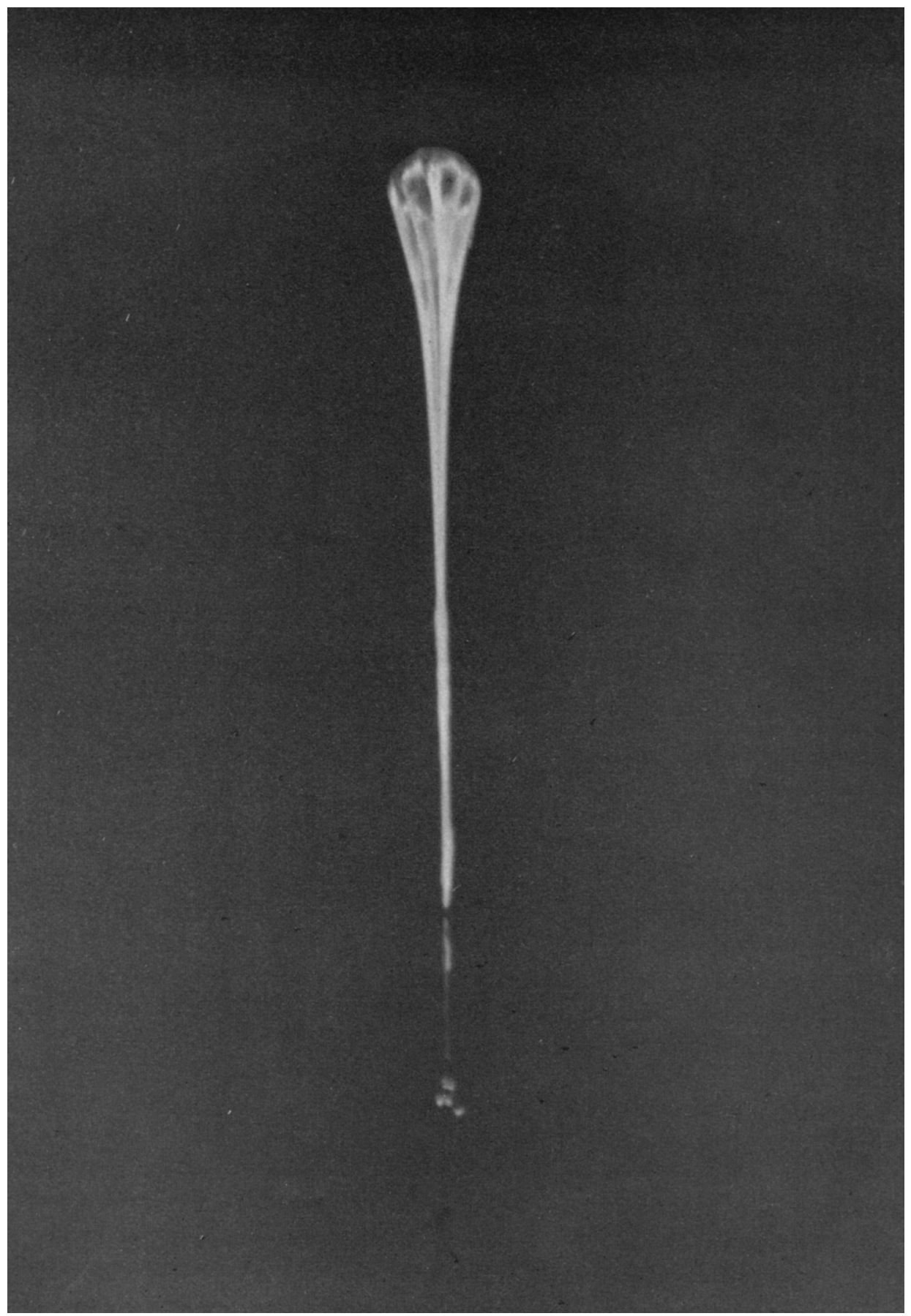

Fig. 12. The same balloon as in Figure 11 rising through the atmosphere carry a high energy gamma ray detector of the Goddard Space Flight Center. 
efficiency of $25 \%$. The energy threshold is about $50 \mathrm{MeV}$. The detector will be continuously pointed away from the Earth and will therefore have no dead time resulting from being pointed at the Earth, but will not have the ability to point at a potential source for long periods. The exposure will be $5 \times 10^{5} \mathrm{~cm}^{2} \mathrm{sr} \mathrm{s} / \mathrm{d}$ and $2 \times 10^{5} \mathrm{~cm}^{2} \mathrm{~s} / \mathrm{d}$ for a source in its scanning path.

\section{CASE TECH GAMMA RAY SPARK CHAMBER}

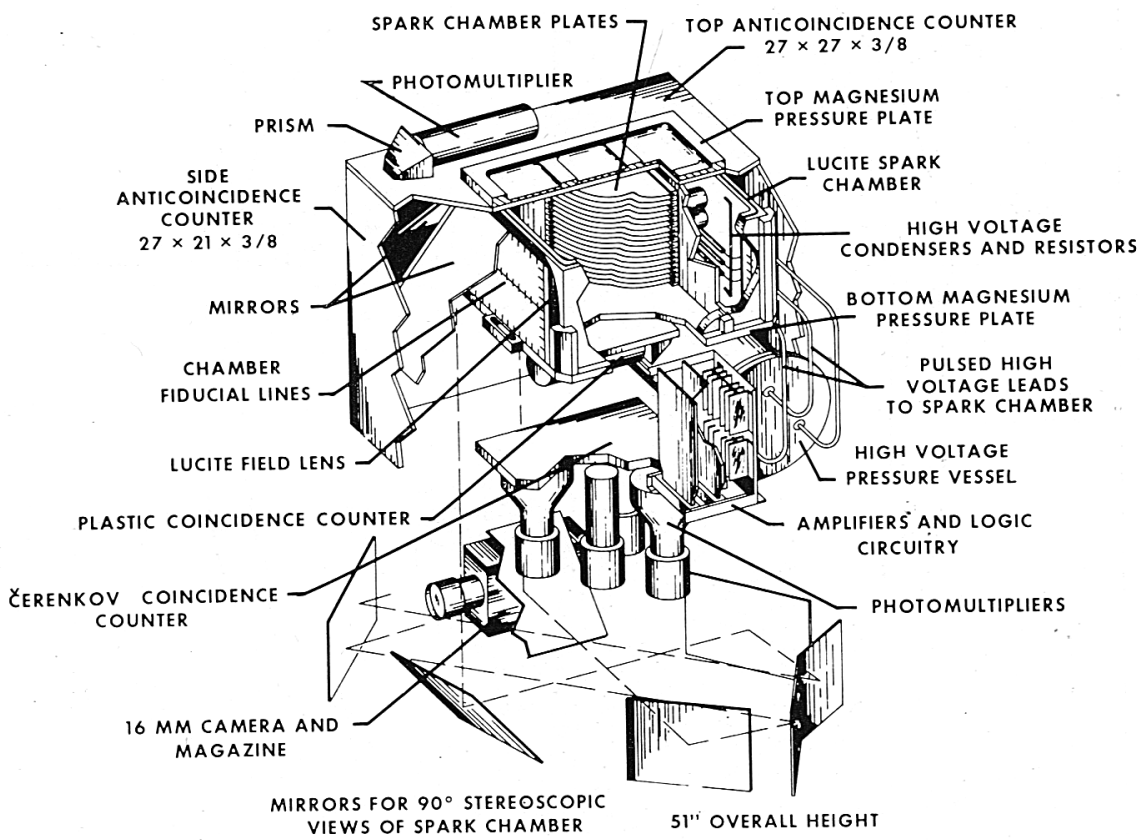

Fig. 13. High energy optical film spark chamber telescope developed by Frye and co-workers at Case.

A somewhat different technique is used in the sonic chamber which uses microphones to record the position of the spark by the use of accurate timing signals. A small version of this type of detector was the first spark chamber in space, being flown on OGO-E by Hutchinson et al. (1969). This instrument is shown in Figure 16.

A sonic spark chamber for a gamma ray telescope was also developed by the Cornell group (Ogelman et al., 1966) and flown successfully on a balloon.

Another gamma ray telescope including a spark chamber has recently been flown on Cosmos 264 by Volobuyev et al. (1969). A 4 gap spark chamber was placed under a one radiation length converter, and one of the main objectives of the spark chamber was to see the shower development.

Magnetic core digitized spark chambers have been developed at Goddard Space 
Flight Center and the Max-Planck-Institut für Extraterrestrische Physik. In both cases, we were guided in the beginning in part by the excellent work of Krienen at CERN (Krienen, 1962 and 1963). The Goddard and Max Planck instruments are similar in basic design. A schematic diagram of the magnetic core spark chamber gamma ray telescope developed by the Max Planck group is shown in Figure 17 (Mayer-Hassel-

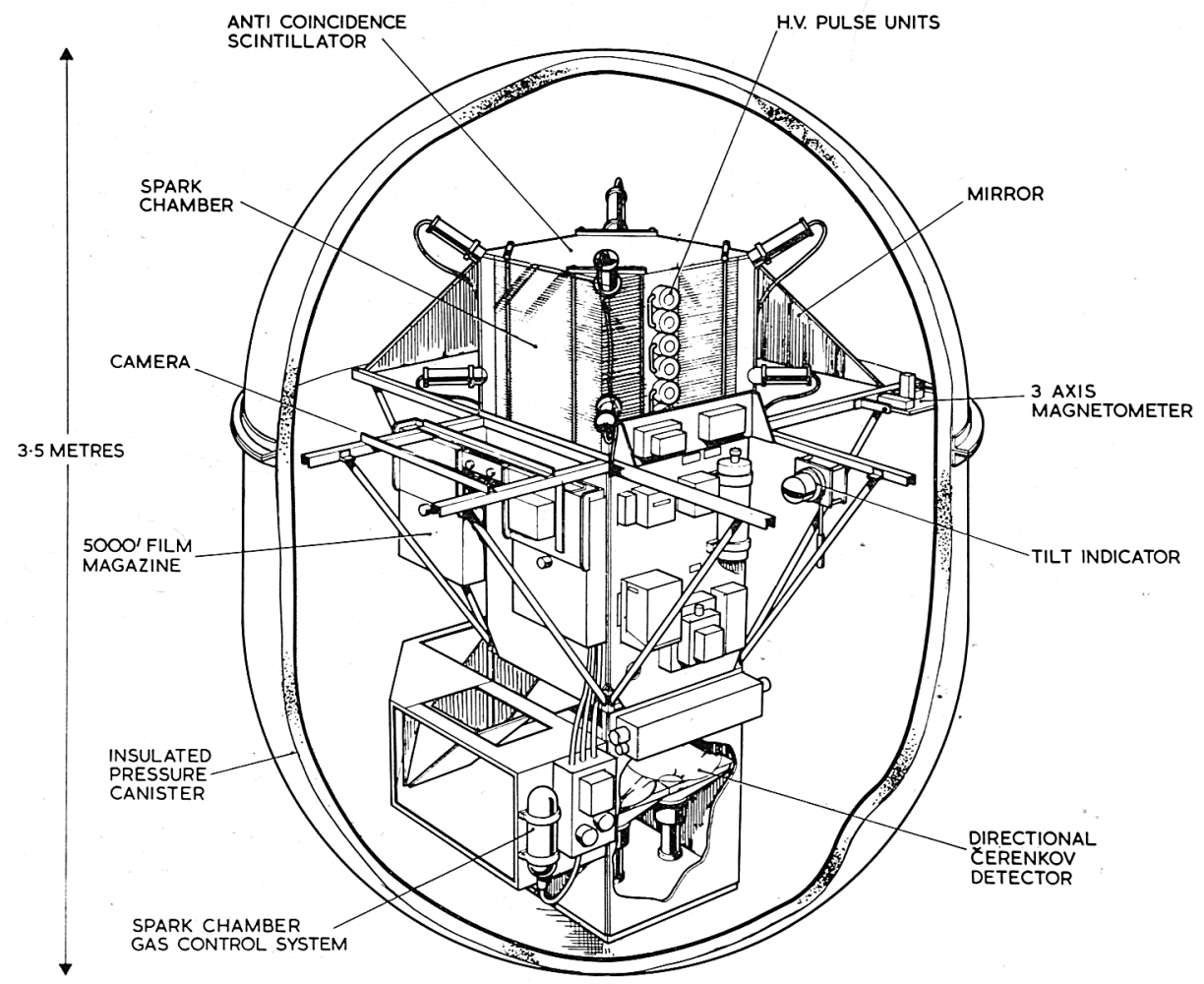

Fig. 14. High Energy optical film spark chamber gamma ray telescope built by Ramsden and co-workers at Southampton.

wander et al., 1970). The second generation $2500 \mathrm{~cm}^{2}$ gamma ray telescope developed at Goddard Space Flight Center (Ross et al., 1969) is shown schematically in Figure 18 and with the dome removed in Figure 19. The GSFC spark chamber itself is shown in Figure 20. For the digitized spark chamber, the flat plates of each module are replaced by two planes of wires orthogonal to each other. One of these grids serves as the ground plane and the other as the high voltage plane. A spark between the planes causes a current to flow in the two orthogonal wires connected electrically by the spark setting cores on the ends of the wire. By reading out the cores a picture can be constructed. Such a picture is shown in Figure 21.

A satellite version of the Goddard detector system will be flown on SAS-B. It is a direct outgrowth of the balloon version flown during 1966 through 1968. except that it 
employs a four telescope system. This approach maintains directionality and adds reliability by increasing redundancy. The SAS-B detector will have the following characteristics: Effective area, $A=500 \mathrm{~cm}^{2}, \Omega=\frac{1}{4} S \cdot \mathscr{h}$.; Efficiency (high energy) $=0.29$; Efficiency $(100 \mathrm{MeV})=0.21$; live time (pointing away from earth $) \simeq 0.9$; fraction of orbit in which detector is not seriously affected by Earth albedo $=\frac{1}{2}$. The net exposures

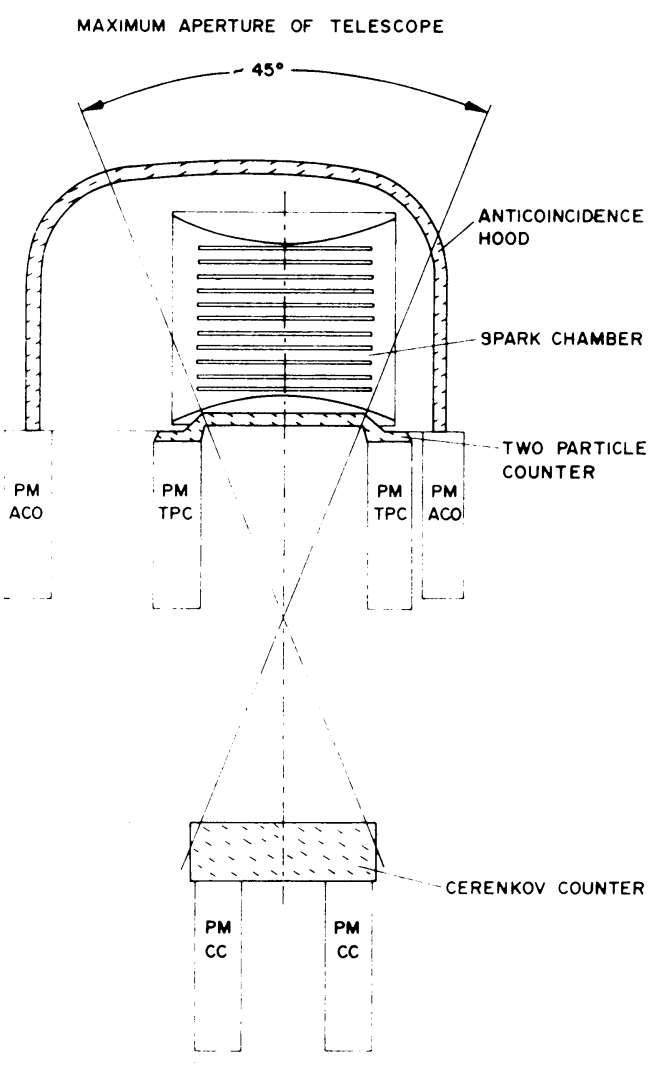

DETECTOR ARRANGEMENT

Fig. 15. High energy gamma ray vidicon spark chamber telescope to be flown on TD-1 by the Max-Planck-Institut für Extraterrestrische Physik, Centre d'Études Nucléaires de Saclay, and the Universita Degli Studi di Milano.

will be $5 \times 10^{6} \mathrm{~cm}^{2} \mathrm{~s} / \mathrm{d}$ and $1.2 \times 10^{6} \mathrm{~cm}^{2} \mathrm{sr} \mathrm{s} / \mathrm{d}$. The angular accuracy is about $1 \frac{1}{2}^{\circ}$ at $100 \mathrm{MeV}$ and varies with energy approximately as $E^{-2 / 3}$. The threshold is not sharp, but is about 20 to $25 \mathrm{MeV}$. The energy of the gamma ray is measured up to about $200 \mathrm{MeV}$. An artist's concept of SAS-B is shown in Figure 22.

It is clear that instruments substantially more sensitive than SAS-B should ultimately be flown. At least two possibilities seem to exist at this time, COS-B and HEAO. For 


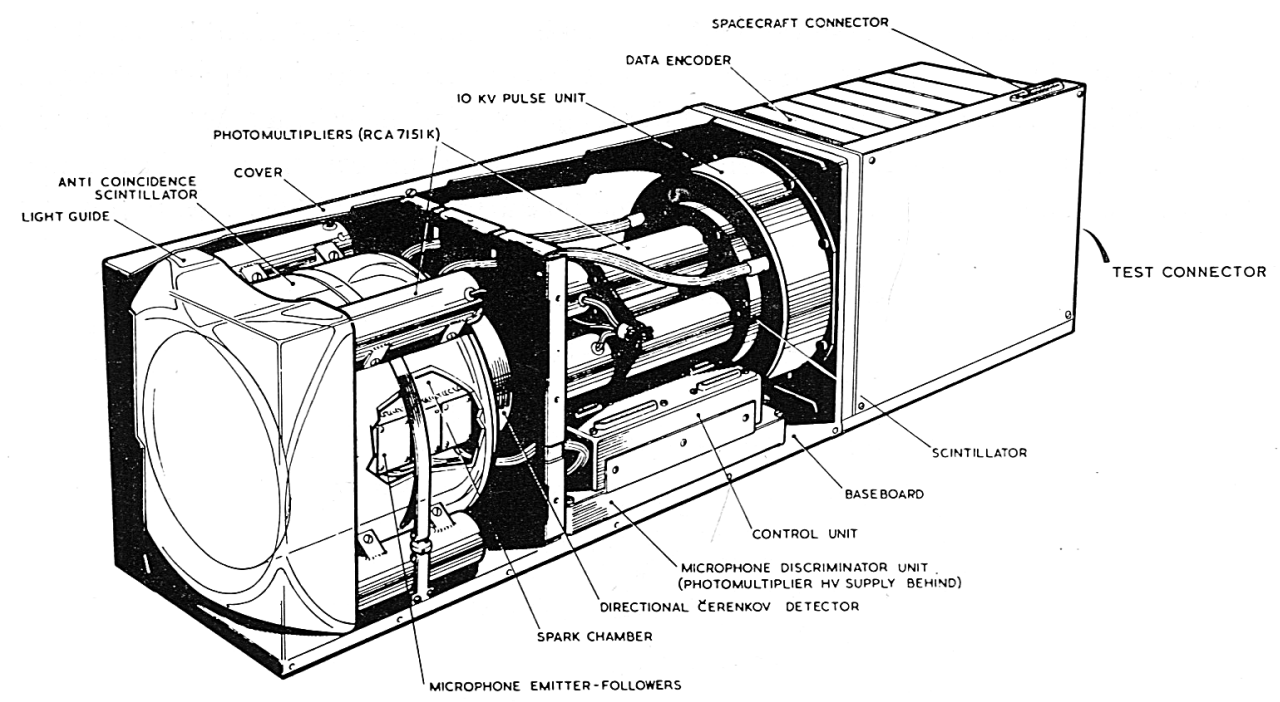

\section{GAMMA-RAY ASTRONOMY EXPERIMENT E-08}

Fig. 16. High energy gamma ray sonic spark chamber flown by Hutchinson et al. (1969) on OGO-E.

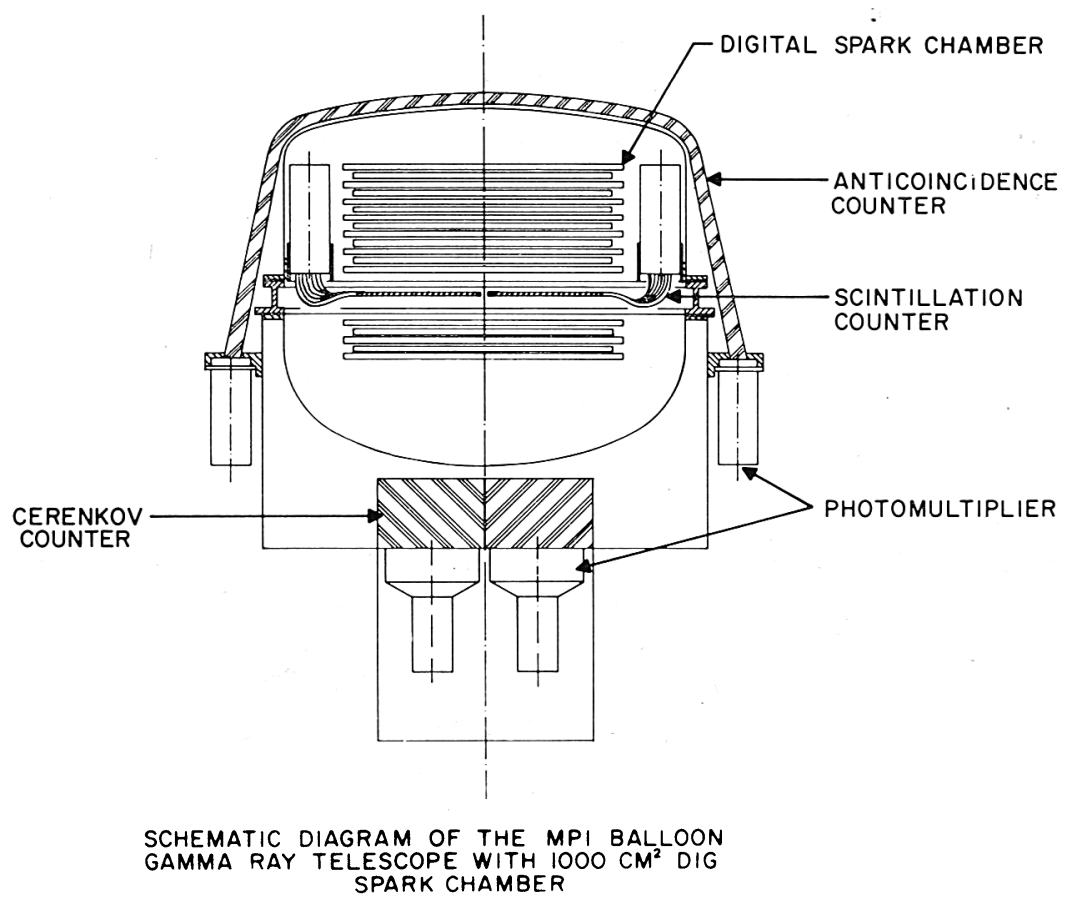

Fig. 17. Schematic diagram of high energy gamma ray magnetic core digitized spark chamber telescope developed at Max-Planck-Institut by Pinkau and Sommer. 
COS- $\mathrm{B}$, it is proposed that a gamma ray detector be the sole experiment on a satellite launched probably into eccentric orbit by a Thor-Delta rocket. It would be a joint effort by Kammerlingh Onnes Laboratory (Leyden), Max-Planck-Institut für Extraterrestrische Physik, Centre d'Études Nucléaires de Saclay, Universita Degli Studi di Milano, and the European Space Laboratory. The instrument would include a series of

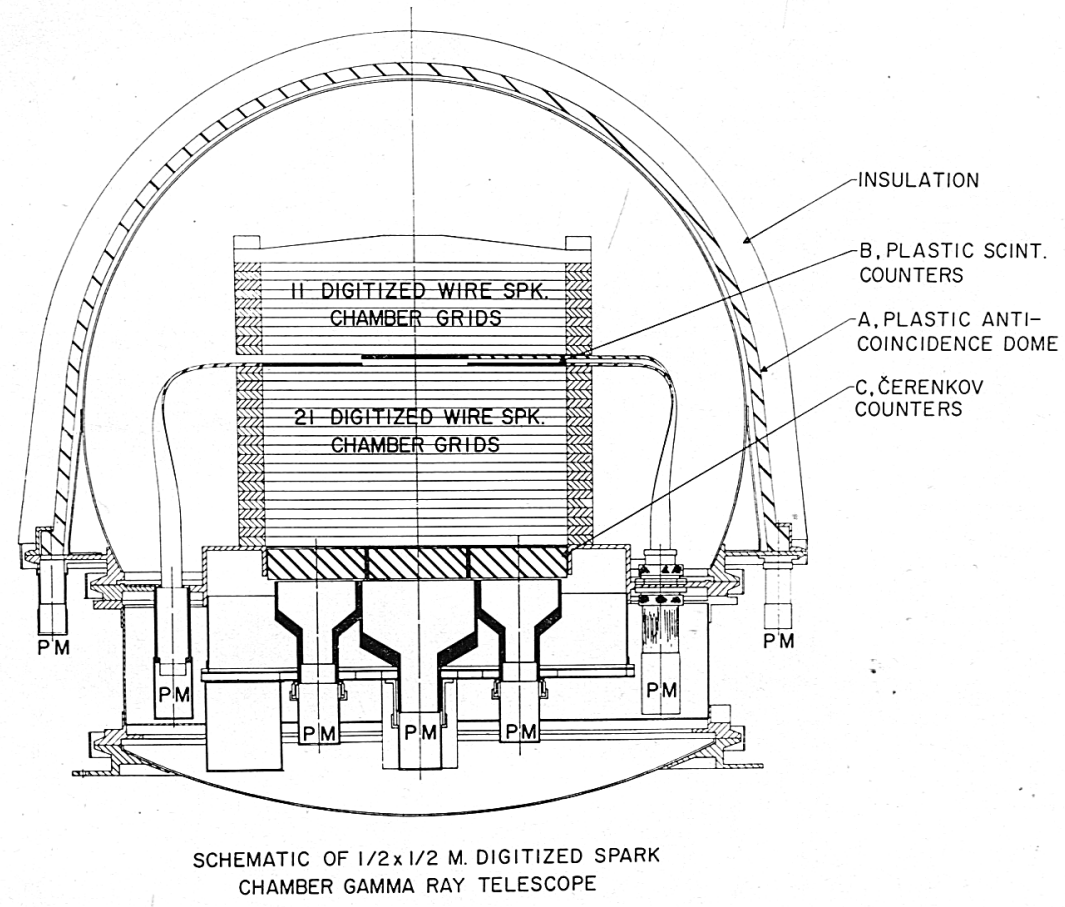

Fig. 18. Schematic design of high energy gamma ray magnetic core digitized spark chamber developed at Goddard by Ehrmann, Fichtel, Kniffen, and Ross.

spark chambers and scintillators surrounded by anticoincidence. The approach and angular resolution would be similar to other spark chamber experiments. The sensitivity would be greater and the energy range on which the energy was determined would be extended to higher energies by using a Cs I crystal of large area. Final approval for HEAO and selection of experiments has not yet occurred, but it is likely that gamma ray experiments will be included.

Nuclear emulsions were used on balloons even before spark chamber by Bracessi et al. (1960); Klarman (1962); Frye et al. (1966); and Fichtel and Kniffen (1965). More recently nuclear emulsions have been combined with spark chambers by May and Waddington (1969) and Kinzer et al. (1969). The latter use a wide gap spark chamber with emulsions as shown in Figure 23. The wide gap spark chamber is used for good angular and spatial resolution with a minimum amount of material after entrance 


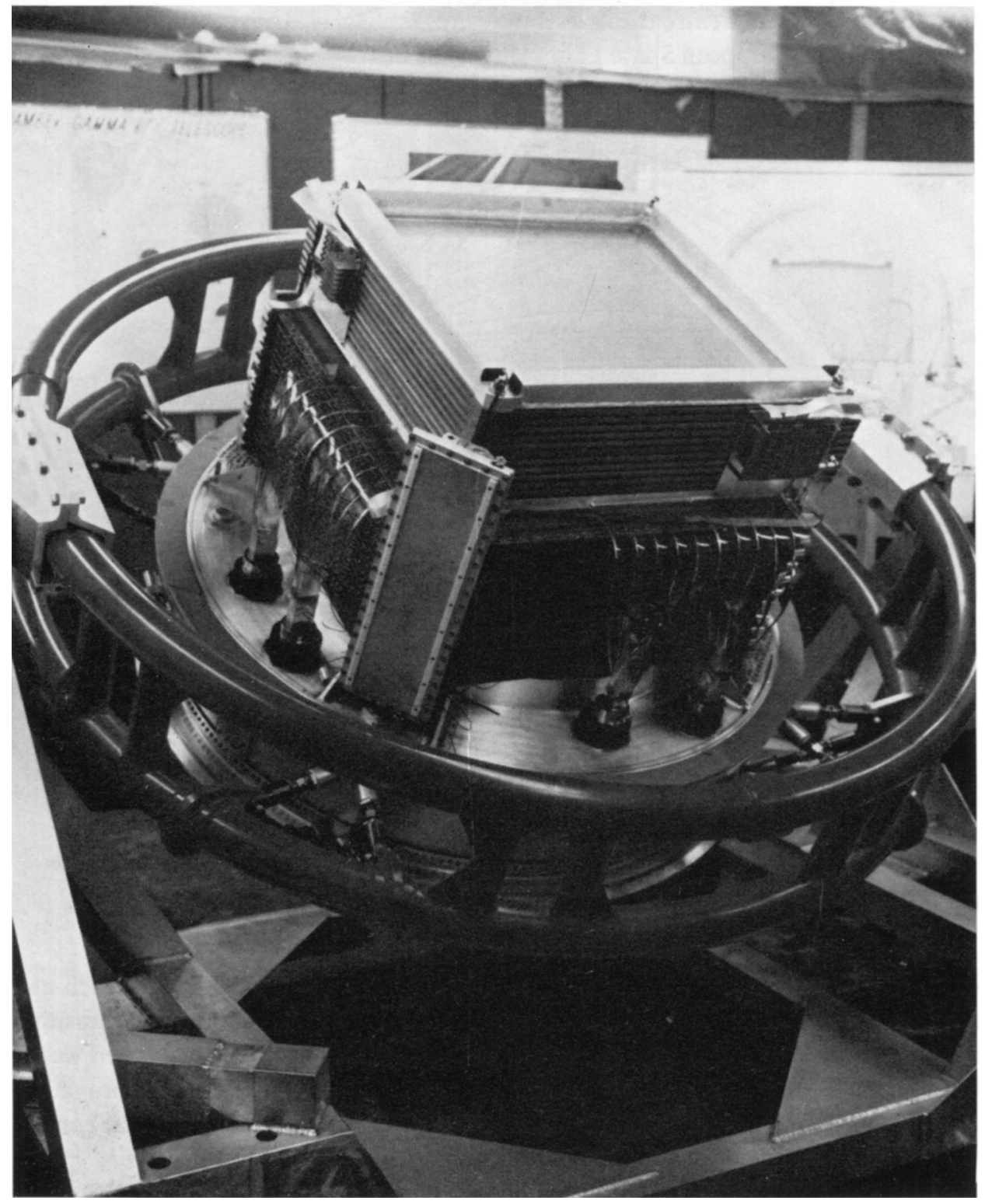

Fig. 19. Balloon gamma ray experiment of Figure 18 with anticoincidence dome removed. 
from the nuclear emulsion. At present charged particle background seems to make this approach unacceptable for satellite use, even if the recovery problem is solved.

In the high energy region above a few hundred $\mathrm{MeV}$, thought is now being given by Fazio and Greisen to using a gas Cerenkov counter either alone or in conjunction with a large spark chamber. Figure 24 shows an example of this approach (Delvaille et al.,

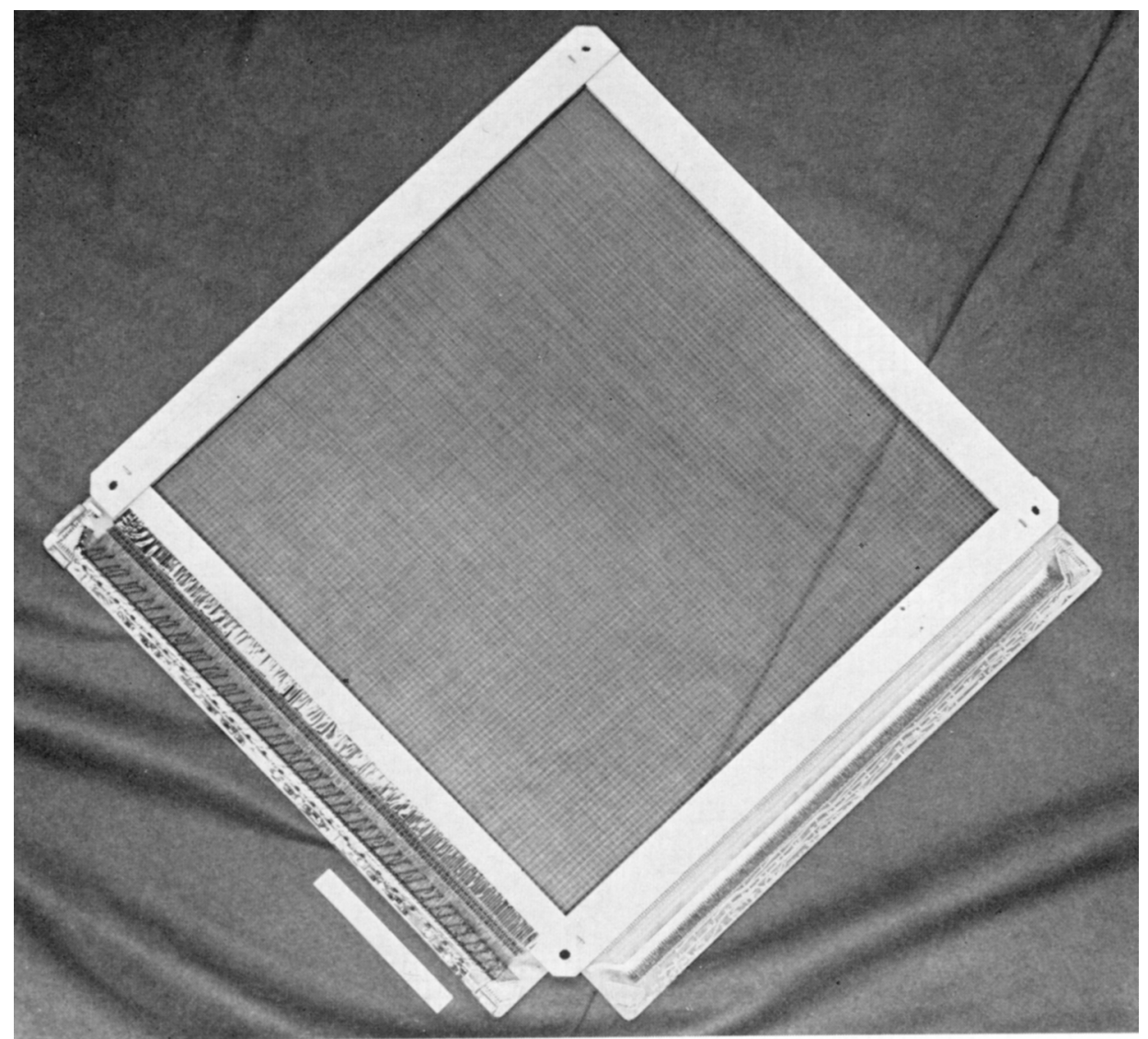

Fig. 20. Individual spark chamber deck of gamma ray telescope shown in Figure 18.

1970). A gas Cerenkov counter records the Cerenkov light produced by a relativistic particle (in this case the negatron and positron formed by the gamma ray) whose velocity exceeds that of the speed of light in the gas. The gas Čerenkov counter has the advantages of fairly high directionality, a relatively low background because of the high energy threshold and the directional property, and the potential for very large collection areas at low cost with little complexity. In this high energy region the electrons scatter relatively little, and. therefore. a relatively thick converter may be used 
after the anticoincidence shield. The pairs pass through a scintillator and then into the low volume Čerenkov gas at angles within a degree or two of their original direction. Helmken and Hoffman (1970) are also giving consideration to applying the gas Čerenkov approach to lower energies.

$x \times \times 339$

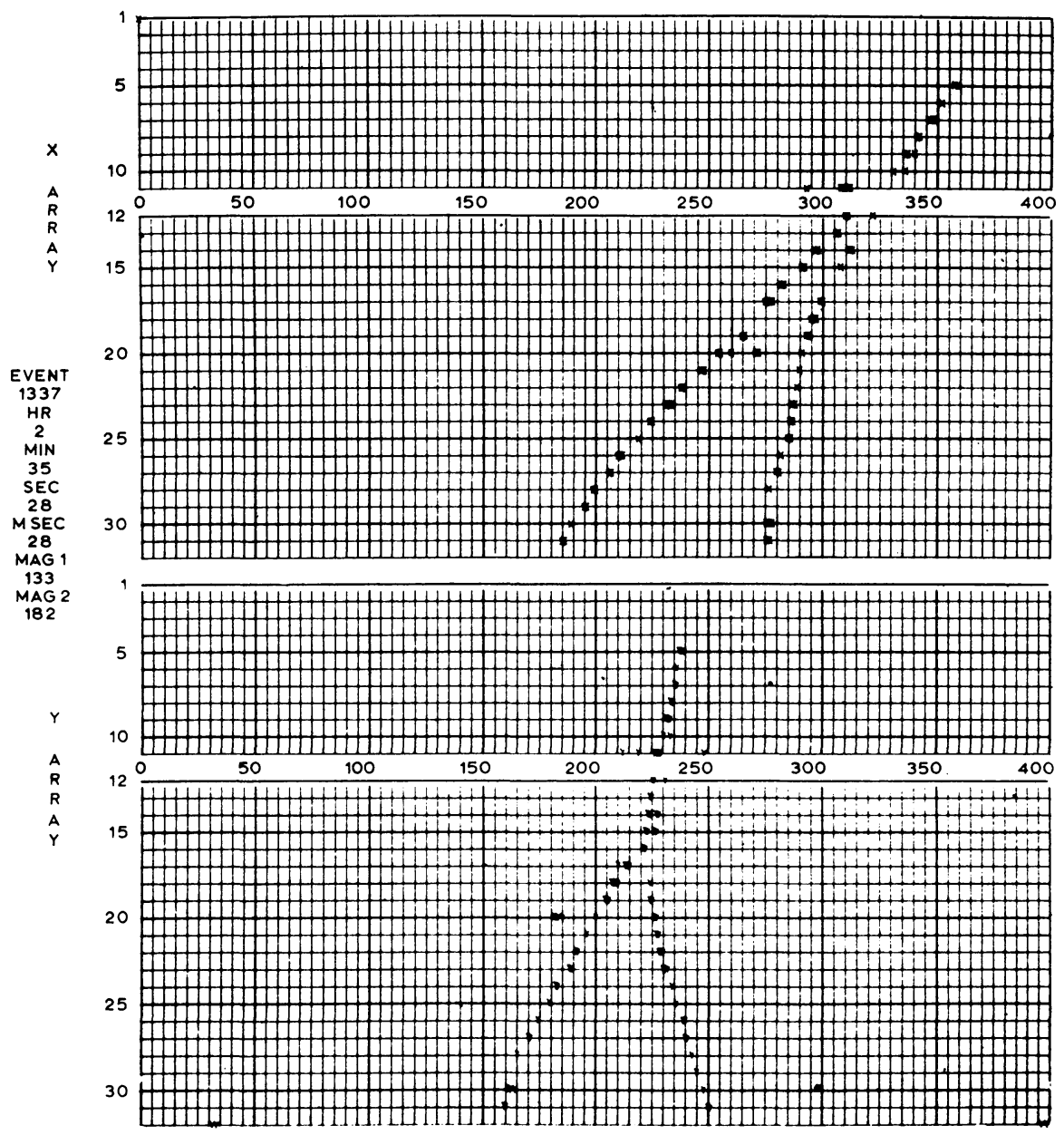

Fig. 21. Computer reproduction of a gamma ray event in the Goddard $2500 \mathrm{~cm}^{2}$ spark chamber telescope.

At extremely high energies (above about $10^{6} \mathrm{MeV}$ ), photons can be detected by instruments at sea level which record the cascade shower particles or the Čerenkov light produced in the atmosphere from a series of interactions initiated by a single incident gamma ray. In the latter case, the negatron and positrons in the shower emit 
Cerenkov light and the direction of the emitted light corresponds very nearly to the direction of the gamma ray as it enters the atmosphere. Figure 25 shows the $10-\mathrm{m}$ optical reflector of Smithsonian Astrophysical Observatory, Mt. Hopkins, Arizona. This approach is complicated by the fact that high energy charged particles also produce cascade showers. A gamma ray source could, however, be detected by the observation of a marked increase in flux from some direction, or by distinguishing between the

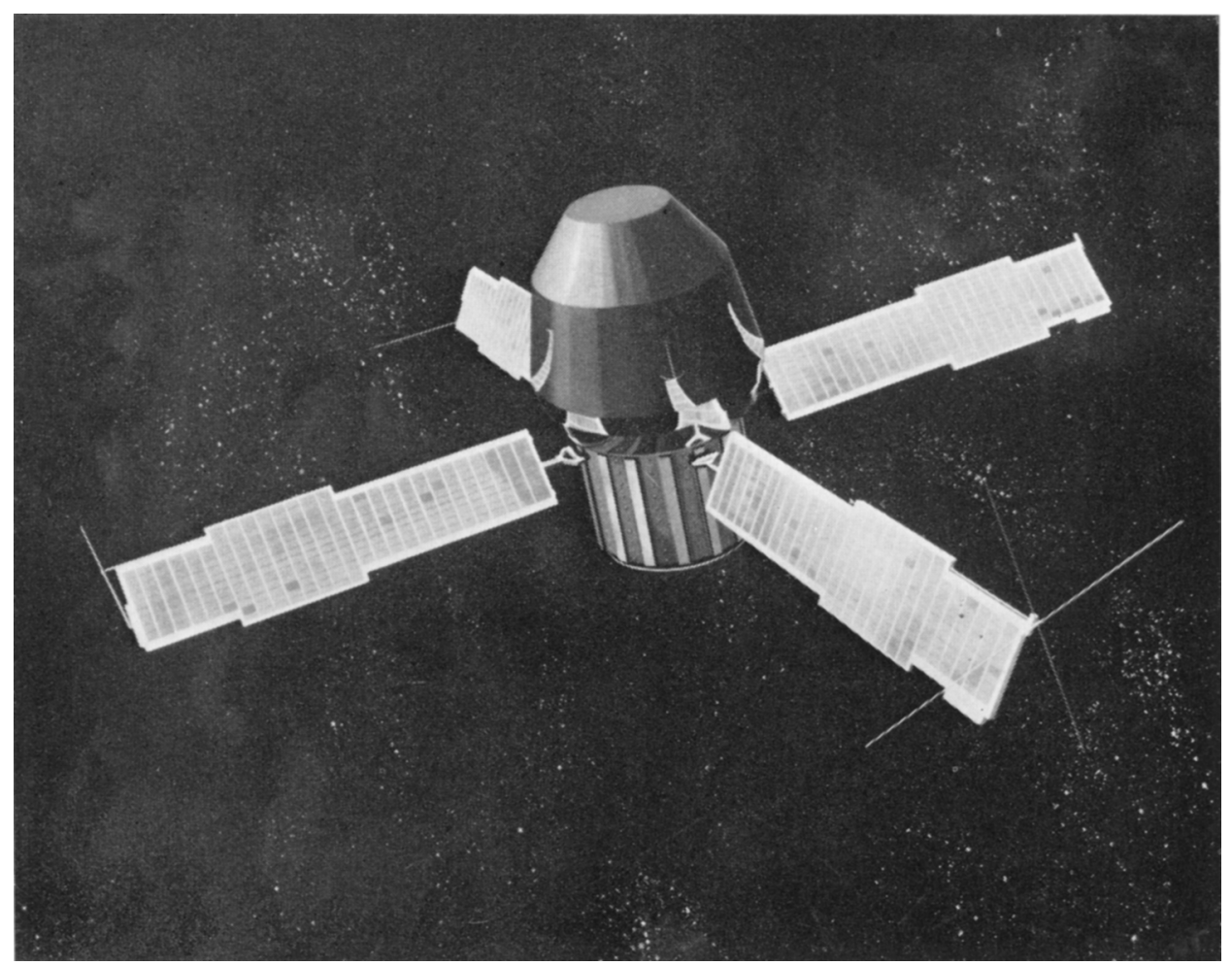

Fig. 22. Artist concept of SAS-B.

types of particle showers as some of the more complex ground instruments may be able to do.

The future hope of high energy gamma ray astronomy appears to lie in getting the large new detectors onto satellites. The size of the system seems to indicate that they must fly as the major experiment on an explorer or together with other experiments on a very large spacecraft. If current plans are realized, gamma ray experiments of this large scale should be flown in the near future. The valuable information on nature's high energy processes should then begin to be revealed in detail. 


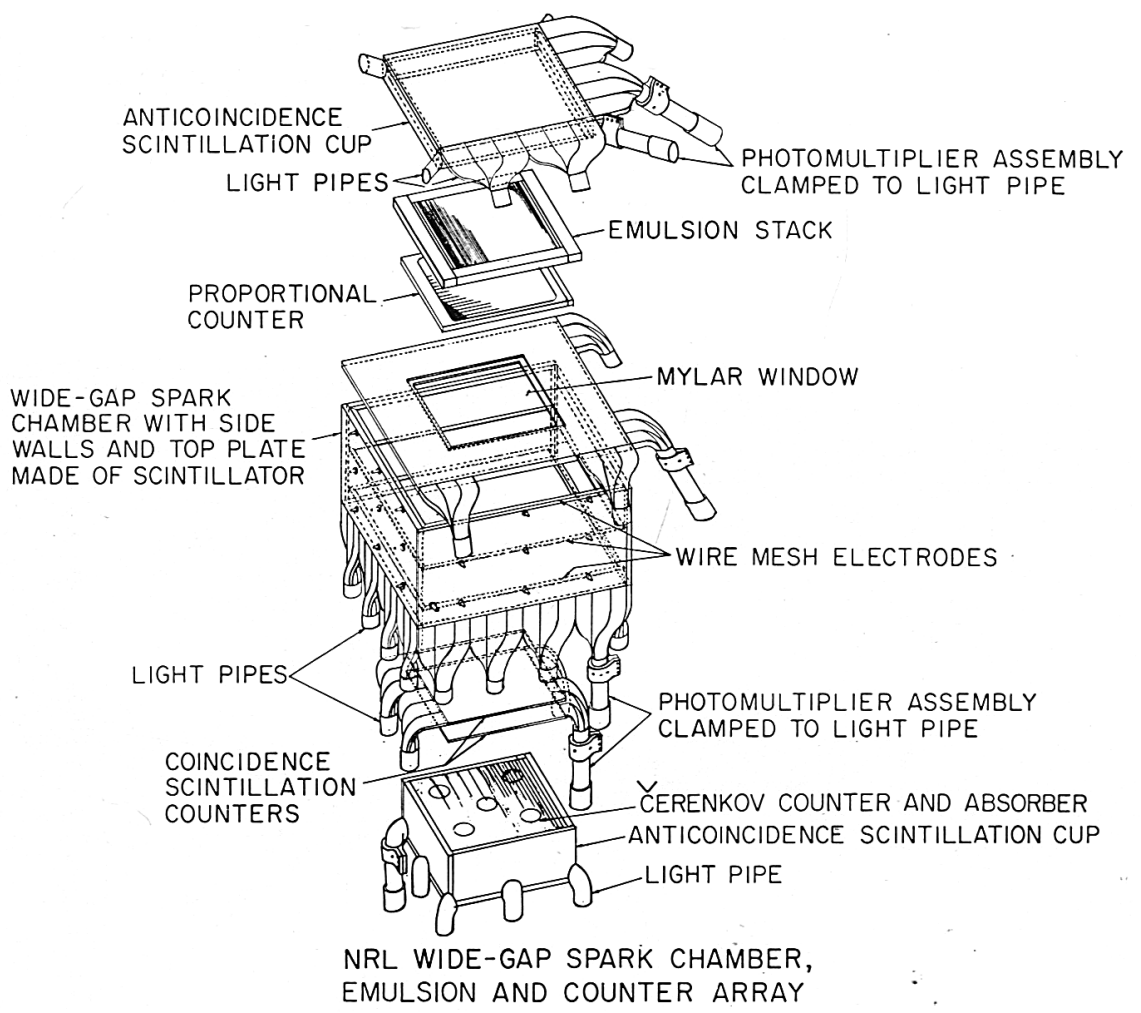

Fig. 23. Wide gap spark chamber used with nuclear emulsions by Kinzer, Seeman, and Share at NRL.

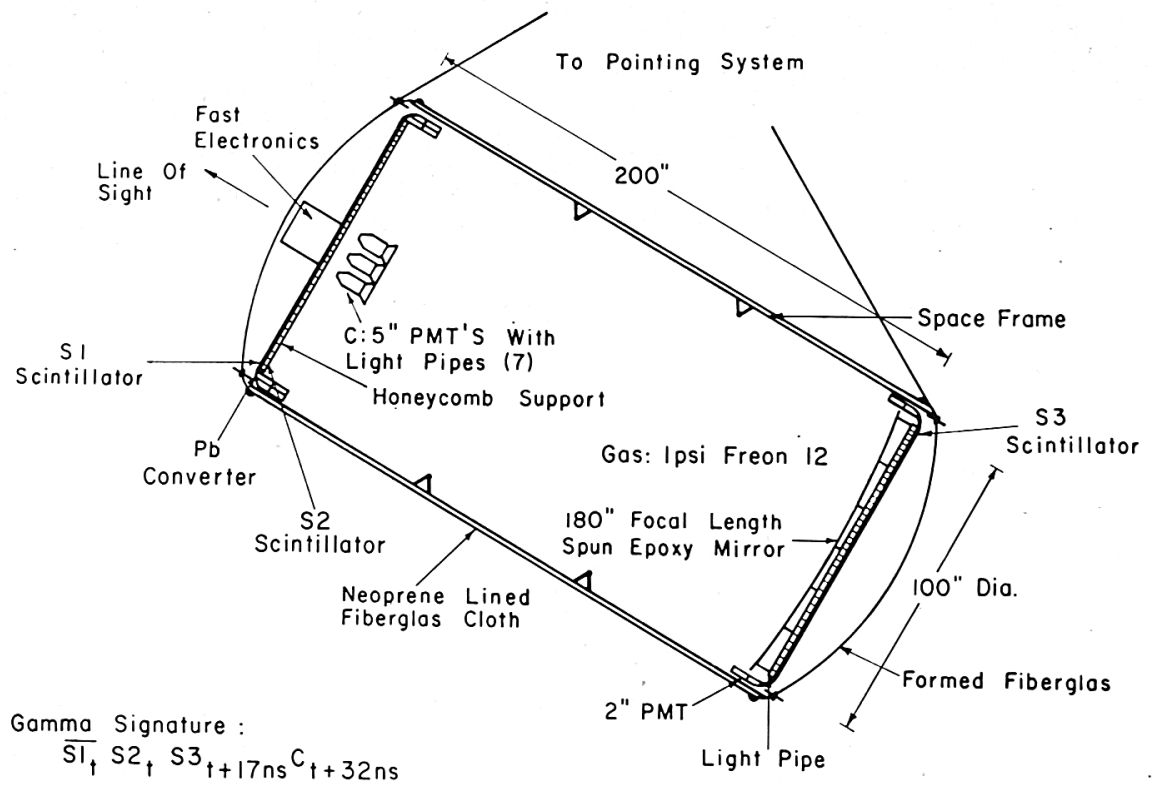

Fig. 24. Large gas Cerenkov counter gamma ray telescope developed by Fazio and Greisen. 


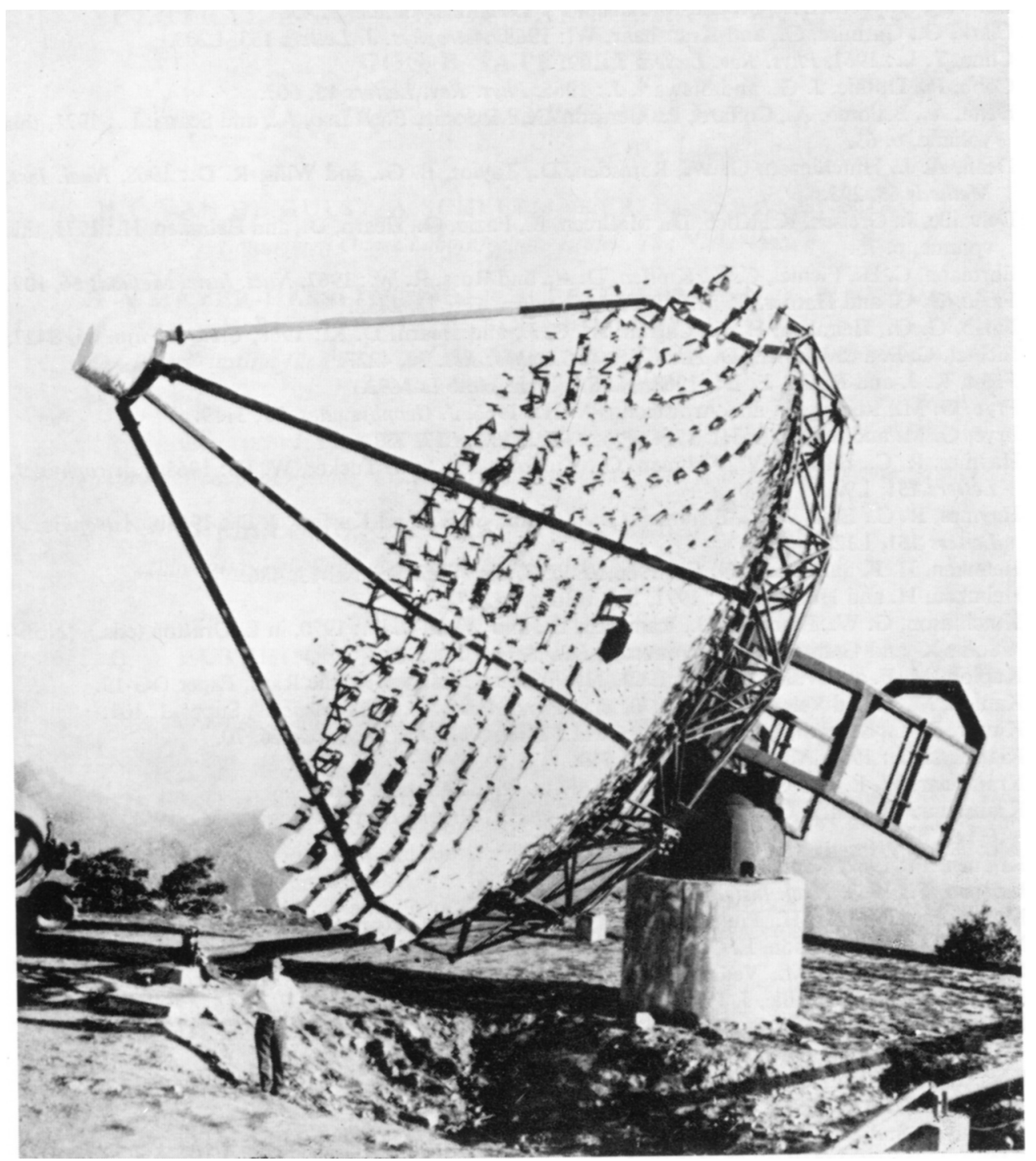

Fig. 25. Ten Meter optical reflector of the Smithsonian Astrophysical Observatory at Mt. Hopkins.

\section{References}

Albernhe, F., Doulade, C., Martin, I. M., Talon, R., and Vedrenne, G.: 1971, this volume, p. 58. Arnold, J. R., Metzger, A. E., Anderson, E. C., and Van Dilla, M. A.: 1962, J. Geophys. Res. 67, 4878. Board, S. J., Dean, A. J., and Ramsden, D.: 1968, Nucl. Inst. Methods 65, 141.

Bracessi, A., Ceccarelli, M., and Salandin, G.: 1960, Nuovo Cimento 17, 691.

Bratolyubova-Tsulukidze, L. I., Grigorov, N. L., Kalinkin, L. F., Melioransky, A. S., Pryakhin, E. A., Savenko, I. A., and Yufarkin, V. Y.: 1969, Paper OG-20, Eleventh International Conference on Cosmic Rays, Budapest, Hungary. Aug. 25-Sept. 4. 
Bui-Van, A., Vedrenne, G., and Mandrou, P.: 1971, this volume, p. 45.

Clark, G., Garmire, G., and Kraushaar, W.: 1968, Astrophys. J. Letters 153, L203.

Cline, T. L.: 1961, Phys. Rev. Letters 7, 109.

Cobb, R., Duthie, J. G., and Stewart, J.: 1965, Phys. Rev. Letters 15, 507.

Dean, A., Bellomo, A., Coffaro, P., Gerardi, G., Madonia, F., Russo, A., and Scarsi, L.: 1971, this volume, p. 63.

Dean, A. J., Hutchinson, G. W., Ramsden, D., Taylor, B. G., and Wills, R. D.: 1968, Nucl. Inst. Methods 65, 293.

Delvaille, J., Greisen, K., Koch, D., McBreen, B., Fazio, G., Hearn, D., and Helmken, H.: 1971, this volume, p. 75.

Ehrmann, C. H., Fichtel, C. E., Kniffen, D. A., and Ross, R. W.: 1967, Nucl. Instr. Methods 56, 109.

Fazio, G. G. and Hafner, E. M.: 1967, J. Geophys. Res. 72, 2452.

Fazio, G. G., Helmken, H. F., Carrah, Jr., S. J., and Hearn, D. R.: 1968, Can. J. Phys. 46, S427.

Fichtel, C. E. and Kniffen, D. A.: 1965, J. Geophys. Res. 70, 4227.

Frost, K. J. and Rothe, E. D.: 1963, NASA Tech. Note D-1693.

Frye, G. M., Reines, F., and Armstrong, A. H.: 1966, J. Geophys. Res. 71, 3119.

Frye, G. M. and Smith, L. H.: 1968, Phys. Rev. Letter's 17, 733.

Haymes, R. C., Ellis, D. V., Fishman, G., Kurfess, J. D., and Tucker, W. H.: 1968a, Astrophys. J. Letters 151, L9.

Haymes, R. C., Ellis, D. V., Fishman, G. J., Glenn, S. W., and Kurfess, J. D.: 1968b, Astrophys. J. Letters 151, L125.

Helmken, H. F. and Fazio, G. G.: 1966, IEEE Trans. Nucl. Sci. NS-13, 486.

Helmken, H. and Hoffman, J.: 1971, this volume, p. 77.

Hutchinson, G. W., Pearce, A. J., Ramsden, D., and Wills, R. D.: 1970, in L. Gratton (eds.), 'NonSolar X-and Gamma-Ray Astronomy', IAU Symp. 37, 300.

Kaplon, M. F. and Valentine, D.: 1969, 11 th Intern. Conf. on Cosmic Rays, Paper OG-19.

Kaplon, M. F. and Valentine, D.: 1970, Acta Phys. Acad. Sci. Hungaricae 29, Suppl. 1, 101.

Kinzer, R. L., Seeman, N., and Share, G. H.: 1969, Nucl. Inst. Methods 76, 70.

Klarmann, J.: 1962, Nuovo Cimento 24, 540.

Kraushaar, W. L. and Clark, G. W.: 1962, Phys. Rev. Letters 8, 106.

Kraushaar, W., Clark, G., Carmire, G., Helmken, H., Higbie, P., and Agogino, M.: 1965, Astrophys. J. 141, 845.

Krienen, F.: 1962, Nucl. Instr. Methods 16, 262.

Krienen, F.: 1963, Nucl. Instr. Methods 20, 168.

Mayer-Hasselwander, H., Pinkau, K., and Sommer, M.: 1970 (private communication).

Metzger, A. E., Anderson, E. C., Van Dilla, M. A., and Arnold, J. R.: 1964, Nature 204, 766.

Niel, M., Cassignol, M., Vedrenne, G., and Bouigue, R.: 1969, Nucl. Inst. Methods 69, 309.

Ogelman, H. B., Delvaille, J. P., and Greisen, K. I.: 1966, Phys. Rev. Letters 16, 491.

Peterson, L. E., Gruber, D., Lewis, S. J., Stone, M. R., and Vette, J. 1.: 1968, Univ. of Calif. at San Diego Report UCSD-SP-68-5.

Ross, R., Ehrmann, C., Fichtel, C., Kniffen, D., and Ogelman, H.: 1969, IEEE Trans. Nucl. Sci. NS-16, 127. Also GSFC X-611-68-443.

Volobuyev, S. A., Galper, A. M., Kirillov-Ugryumov, V. G., Luchkov, B. I., Yu, Ozerov, V., Rozental, I. L., Shermanzon, E. M., Grigorov, N. L., Kelinkin, L. F., Malioransky, A. S., Savenko, I. A., and Shashko, T. A.: 1969, 11 th Intern. Conf on Cosmic Rays, Paper OG-20.

\section{DISCUSSION}

K. Pinkau: Are there any figures of merit available for the low energy telescopes? What is their ability to collimate gamma rays and to reject background like neutrons?

C. E. Fichtel: The ability to collimate gamma rays and still maintain a high sensitivity depends to a large degree on the thickness of the collimator and hence the weight capability of the vehicle. The rejection of neutrons can be enhanced somewhat by pulse shape discrimination. I have no specific details on figures of merit except the detection limits shown in Figure 6 for the OSO-1 experiment. In general, the background calibrations are meaningful only when the experiment is placed in the environment that it will be used to collect data. 